\title{
IMPLEMENTATION OF A SPECTROPHOTOMETER TEACHING AND LOW COST: APPLICATION IN PHYSICS AND CHEMISTRY LABORATORIES FAFISM
}

Kátia Isabel Milagres ${ }^{1 *}$; Camila Rodrigues Amaral$^{2}$; Érica M. da Silva Santos ${ }^{2}$; Tarcísio B. Marques ${ }^{2}$; Guilherme de O. Gomes ${ }^{1}$

${ }^{1}$ Acadêmicos do Curso de Licenciatura em Química - FAFISM;

${ }^{2}$ Professores da FAFISM

*(isabelmilagre@yahoo.com.br).

Resumo: A fotometria no campo visual possui ampla aplicação em laboratórios de análises e pesquisas físicas, químicas, bioquímicas e farmacológicas. É fundamental que estudantes de Física e Química possuam conhecimento sobre esta técnica. Um entrave para a ampla utilização dessa técnica é o custo dos equipamentos e dos materiais a serem utilizados. O objetivo deste trabalho foi à introdução de um fotômetro didático, cuja construção e técnica com baixo custo operacional e de fácil utilização e produção de resultados, se coloque como solução prática para aprimoramento das aulas. As características destacadas no trabalho fazem do fotômetro proposto uma ferramenta alternativa economicamente viável para análises químicas nos laboratórios da Faculdade para pesquisa e/ou ensino.

Palavras-chave: Fotômetro, Aparato, Ludke, Absorção

Abstract: The photometry in the visual field has wide application in analytical laboratories and research physical, chemical, biochemical, pharmacological, etc. It is essential that students of physics and chemistry have knowledge about this quantitative technique. One obstacle to the widespread use of this technique is the cost of equipment and materials to be used. The objective of this work is the introduction of a photometer didactic, whose construction and technique with a low operating cost and the easy using and producing results, as a practical solution to put school improvement. The features highlighted in the work do the proposed photometer an economically viable alternative tool for chemical analysis in the laboratories of the Faculty for research and / or teaching.

Keywords: Photometer, Apparatus, Ludke, Absorption 


\section{INTRODUÇÃO}

A atividade experimental realizada em laboratório é fundamental para o ensino de Química e física e a realização de práticas experimentais podem ser direcionadas para atingir vários objetivos, tais como aplanamento de aprendizagem, habilidades motoras, hábitos, técnicas e manejo de aparelhos, aprendizagem de conceitos e suas relações, leis e princípios.

Entretanto, um dos grandes desafios do uso de aulas experimentais no ensino de Química e áreas afins é construir um elo entre o conhecimento ensinado e o cotidiano dos estudantes. A ausência de conexão e contextualização entre o conteúdo passado em sala de aula e o dia-a-dia, é um motivo para justificar a indiferença entre os alunos e também em relação aos próprios docentes quando do uso da experimentação.

Atividades práticas e experimentais têm como finalidade desenvolver não somente pesquisas científicas, como também desenvolver a compreensão de conceitos ou colocá-los no momento adequado para que os alunos percebam sua relação com a teoria vista em sala de aula. A forma como se utiliza o laboratório é mais importante do que a própria prática experimental em si, sendo que a concordância dos alunos de aulas experimentais está muito ligada a este fato.

A fotometria no campo visual possui ampla aplicação em laboratórios de análises e pesquisas físicas, químicas, bioquímicas e farmacológicas. Inúmeras vantagens contribuem para sua popularidade; a principal é o fato de ser uma técnica quantitativa. Fotômetros simples, portáteis e de baixo custo, que empregam LED como fonte de radiação têm sido desenvolvidos e usados em aplicações analíticas [1-4].

Diante disto é fundamental que estudantes de Física e Química possuam conhecimento sobre esta técnica. Um entrave para a ampla utilização dessa técnica é o custo dos equipamentos e dos materiais a serem utilizados. Daí, a introdução do fotômetro didático, cuja construção e técnica com baixo custo operacional e de fácil utilização e produção de resultados, se coloca como solução prática para aprimoramento das aulas.

Muitas das inovações referentes à espectrofotometria consistem em estratégias para aumento de sensibilidade, visando ampliação da faixa de aplicação da técnica e permitindo, em alguns casos, que medidas em concentrações da ordem de nmol. $\mathrm{L}^{-1}$ sejam efetuadas [2]. O presente trabalho possibilita o aluno entender a constante de proporcionalidade que relaciona a absorbância com o caminho óptico e a concentração das espécies e a determinar os princípios fundamentais da espectroscopia de absorção da radiação.

O aparato experimental desenvolvido teve como objetivo possibilitar estudos qualitativos e quantitativos de vários aspectos experimentais relacionados às técnicas de fotometria, principalmente a comprovação experimental da Lei de Lambert-Beer, que é difícil de ser medida diretamente em equipamentos comerciais [5]. A repetitividade e reprodutibilidade desse experimento justifica sua adoção na prática de ensino para as licenciaturas em Física, Química e Biologia, onde dentre vários motivos destacamos a importância da prática experimental na formação do professor, as limitações de tempo e orçamento, agravantes na qualidade do ensino em ambientes interdisciplinares. 
Tal aparato experimental pode ser aplicado não somente nos cursos de licenciatura, mas também em cursos de bacharelado e áreas afins, o presente trabalho teve seu foco principal na aplicação em cursos de licenciatura.

\section{PROCEDIMENTO ESPERIMENTAIS}

\section{Funcionamento do Fotômetro}

O aparato experimental desenvolvido neste trabalho foi montado a partir do modelo desenvolvido por Ludke [5] onde em uma matriz de contato foi anexado um fototransístor empregado como sensor de luz e ligado a um amplificador operacional TL081 de variável entre 1 e 10 vezes em modo amplificador D.C., para aumentar a precisão da leitura na voltagem. A medida da tensão de saída em volts mostrada em um multímetro digital é proporcional à intensidade da luz captada pelo sensor. Assim a medida do multímetro indicará a intensidade do sinal de uma forma linear.

Sobre o fototransístor foi colocado um tubo de vidro de $10 \mathrm{~mm}$ de diâmetro e $20 \mathrm{~cm}$ de altura, fixado por um suporte. Em um segundo tubo de $5 \mathrm{~mm}$ de diâmetro e móvel, foi afixado o LED transístor, este conectado a uma fonte de $+12 \mathrm{~V}$ e resistor de $820 \Omega$, Figura 1. Para as medidas de absorção em comprimentos de onda no visível, para fins de determinação da lei de Lambert-Beer foi utilizado o LED verde e vermelho.

Para obter um resultado mais preciso foi necessário minimizar a luz ambiente sobre o aparato. Para isso foi construído uma câmera escura de papelão ao seu redor, não permitindo gerar medidas incorretas da intensidade da luz.

\section{Figura 1}

\section{Medidas analíticas}

As medidas foram obtidas a partir da variação da distância entre o LED e o sensor, que pôde ser medida com uma régua de $15 \mathrm{~cm}$ de escala e os sensores foram afastados entre si. A medida da tensão de saída em volts mostrada em um multímetro indica a intensidade do sinal de uma forma linear. Para avaliar o desempenho do fotômetro proposto, realizaram-se as análises e o aparato experimental forneceu medidas de absorção em comprimento de onda no campo visual para duas soluções distintas de Permanganato de Potássio $\left(\mathrm{KMnO}_{4}\right)$ e Sulfato de Cobre $\left(\mathrm{CuSO}_{4}\right)$, ambos nas concentrações 0,$5 ; 1,0 ; 1,5 ; 2,0 ; 2,5 \times 10^{-4}$ mol. $\mathrm{L}^{-1}$. Essas soluções foram escolhidas devido à capacidade de absorção e por estarem dentro da faixa de análise do fotômetro.

As medidas de valores relativos de atenuação da luz captada pelo sensor I(x) expressa em dB é em função da distância entre o LED e o sensor, que pode ser medida com uma régua de $15 \mathrm{~cm}$ de escala. Assim 
se $\mathrm{I}_{0}$ for a intensidade máxima da luz em $\mathrm{x}=0$, a intensidade relativa é dada por $\mathrm{I}_{\mathrm{dB}}=10 \log \left[\mathrm{I}(\mathrm{x}) / \mathrm{I}_{0}\right]$ e o gráfico $\mathrm{I}_{\mathrm{dB}} \mathrm{vS} \mathrm{x}$, fornece o coeficiente de extinção molar $\left(\varepsilon_{\lambda}\right)$ da solução [6].

\section{RESULTADOS E DISCUSSÕES}

Os dados medidos no equipamento são referentes à voltagem medida no multímetro, a partir dos valores obtidos. As tabelas 1 e 2 foram produzidas com os valores de intensidade luminosa absorvida $\operatorname{IdB}=$ $10 \log [\mathrm{I}(\mathrm{x}) / \mathrm{I} 0]$.

\section{Tabelas 1 e 2}

A figuras 2 e 3 apresentam as curvas $I d B$ vs $x$ para diferentes concentrações de $\mathrm{CuSO}_{4}$ e $\mathrm{KMnO}_{4}$ respectivamente. As medidas de absorbância para as soluções em concentrações diferentes foram determinadas usando a Lei de Lambert-Beer, $\mathrm{A}=-0,1 \mathrm{I}_{\mathrm{dB}}=\varepsilon_{\lambda} \mathrm{Cl}$, que é o princípio matemático para medidas de absorção de radiação em amostras nos estados sólido, líquido e gasoso, nas regiões visível, ultravioleta e infravermelho do espectro eletromagnético [7].

\section{Figuras 2 e 3}

O coeficiente de absorbância das soluções foi obtido a partir do gráfico A vs C apresentados na Fig.4 e Fig. 5, tendo sido encontrados os seguintes valores: $\mathrm{KMnO}_{4}=0,05121 \pm 0,01431 \mathrm{~L} / \mathrm{cm}^{3} \mathrm{~mol}$ e para o $\mathrm{CuSO}_{4}=0,01496 \pm 0,00279 \mathrm{~L} / \mathrm{cm}^{3} \mathrm{~mol}$, resultados com precisão satisfatória.

\section{Figuras 4 e 5}

\section{CONCLUSÃO}

O fotômetro proposto mostrou-se uma alternativa viável e de potencial para o estudo de interações nas disciplinas de bioquímica e biofísica em caráter didático pedagógico. É uma ferramenta alternativa economicamente viável para as análises químicas nos laboratórios da Faculdade para a pesquisa e/ou ensino.

Esse sistema possui manutenção simples e barata, pois utiliza componentes baratos e facilmente encontrados, além de uma eletrônica simples. O custo do aparato foi de $\mathrm{R} \$ 20,00$, um valor bem inferior a um equipamento comercial simples de aproximadamente $\mathrm{R} \$ 2500$.

Ao final deste trabalho foi possível verificar que o sistema desenvolvido apresentou-se eficiente para aplicações didáticas, tendo em vista que sua aplicação é em caráter de experimentação utilizada em laboratório didático, onde as atividades práticas possam ser consideradas eficazes como facilitadoras no 
processo de ensino e aprendizagem, as mesmas devem propiciar uma maior motivação na sua realização por parte do aluno, despertando seu interesse em participar do processo de aprendizagem.

Devido a sua simplicidade, o mesmo apresenta limitações técnicas, o que inviabiliza sua utilização para fins de pesquisa experimental de alto nível, desta forma o mesmo não foi comparado com um fotômetro comercial.

\section{REFERÊNCIAS}

[1] NERY, A. L. P; FERNANDEZ, C. Fluorescência e estrutura atômica: Experimentos simples para abordar o tema. Química nova na escola, n.19, p. 39-42, 2004.

[2] ROCHA, F. R. P; TEIXEIRA, L. S. G. Estratégias para aumento de sensibilidade em espectrofotometria UV-VIS. Química nova, v.27, n.5, p.807-812, 2004.

[3] GAIÃO, E. N; MEDEIROS, E.P; LYRA, W.S; MOREIRA, P.N.T; VASCONCELOS, P.C; SILVA, E.C; ARAÚJO, M.C.U. Um fotômetro multi-led microcontrolado, portátil e de baixo custo. Química Nova, v.28, n.6, p.1102-1105, 2005.

[4] ARAUJO, M.C.U; SANTOS, S.R.B; SILVA, E.A; VÉRAS, G. Um fotômetro de fluxo para análises clínicas a base de um diodo emissor de luz bicolor. Química nova, v.20, n.2, p.137-145, 1997.

[5] LÜDKE, E. Um espectrofotômetro de baixo custo para laboratório de ensino: aplicações no ensino da absorção eletrônica e emissão de fluorescência. Revista Brasileira de Ensino de Física, v. 32, n. 1, p.15061508, 2010.

[6] ATKINS, P.W.; PAULA, J. Physical Chemistry (Ox-ford University Press, Oxford), 8th ed., p. 431-508, 2006.

[7] GALO, A.L; COLOMBO, M.F. Espectrofotometria de longo caminho óptico em espectrofotômetro de duplo-feixe convencional: uma alternativa simples para investigações de amostras com densidade óptica muito baixa. Química Nova, v.32, n. 2, p.488-492, 2009. 


\section{LISTA DE TABELAS:}

Tabela 1- Medidas de Intensidade Luminosa vs distância para a solução de $\mathrm{CuSO}_{4}$

Tabela 2 - Medidas de Intensidade Luminosa vs distância para a solução de $\mathrm{KMnO}_{4}$

\section{LISTA DE FIGURAS:}

Figura 1- Fotômetro didático desenvolvido no laboratório da FAFISM.

Figura 2 - Intensidade Luminosa vs distância para diferentes concentrações de $\mathrm{CuSO}_{4}$

Figura 3 - Intensidade Luminosa vs distância para diferentes concentrações $\mathrm{KMnO}_{4}$

Figura 4- Dados Experimentais para a espectroscopia do $\mathrm{CuSO}_{4}$ para diferentes concentrações molares utilizando o LED emissor verde

Figura 5- Dados Experimentais para a espectroscopia do $\mathrm{KMnO}_{4}$ para diferentes concentrações molares utilizando o LED emissor vermelho. 


\begin{tabular}{|r|r|r|r|r|r|}
\hline \multicolumn{1}{|l|}{ Distância } & \multicolumn{5}{|c|}{ IdB } \\
\hline $\mathbf{m m}$ & \multicolumn{5}{|c|}{ Concentração } \\
\cline { 2 - 7 } & \multicolumn{5}{|c|}{} \\
\hline $\mathbf{0 , 5}$ & $-4,15$ & $-5,44$ & -10 & $-5,64$ & $-7,78$ \\
\hline $\mathbf{1}$ & $-4,15$ & $-4,47$ & $-6,99$ & $-7,4$ & $-6,02$ \\
\hline $\mathbf{1 , 5}$ & $-3,36$ & $-8,45$ & $-6,99$ & $-7,4$ & $-6,02$ \\
\hline $\mathbf{2}$ & $-2,69$ & $-3,68$ & $-5,23$ & $-5,64$ & $-4,77$ \\
\hline $\mathbf{2 , 5}$ & $-2,11$ & $-3,01$ & $-3,99$ & $-4,39$ & $-4,47$ \\
\hline $\mathbf{3}$ & $-1,6$ & $-2,43$ & $-3,01$ & $-3,42$ & $-3,01$ \\
\hline $\mathbf{3 , 5}$ & $-1,14$ & $-3,01$ & $-2,22$ & $-3,42$ & $-3,01$ \\
\hline $\mathbf{4}$ & $-1,14$ & $-3,01$ & $-2,22$ & $-1,44$ & $-2,34$ \\
\hline $\mathbf{4 , 5}$ & $-0,726$ & $-3,68$ & $-1,55$ & $-3,42$ & $-2,34$ \\
\hline $\mathbf{5}$ & $-0,348$ & $-2,43$ & $-0,969$ & $-1,44$ & $-1,76$ \\
\hline
\end{tabular}

Tabela 3- Medidas de Intensidade Luminosa vs distância para a solução de $\mathrm{CuSO}_{4}$

\begin{tabular}{|r|r|r|r|r|r|}
\hline \multicolumn{1}{|l|}{ Distância } & \multicolumn{5}{|c|}{ IdB } \\
\hline $\mathbf{m m}$ & \multicolumn{5}{|c|}{ Concentração $^{-2}$} \\
\cline { 2 - 6 } & \multicolumn{5}{|c|}{$\mathbf{2}$} \\
\hline $\mathbf{x}$ & 0,5 & 1 & 1,5 & 2,5 \\
\hline $\mathbf{0 , 5}$ & $-5,71$ & $-0,263$ & $-0,17$ & $-0,817$ & $-0,263$ \\
\hline $\mathbf{1}$ & $-3,22$ & $-0,544$ & $-0,17$ & $-0,67$ & $-0,544$ \\
\hline $\mathbf{1 , 5}$ & $-2,81$ & $-0,843$ & $-0,348$ & $-0,527$ & $-0,843$ \\
\hline $\mathbf{2}$ & $-2,08$ & $-1,16$ & $-0,725$ & $-0,389$ & $-1,16$ \\
\hline $\mathbf{2 , 5}$ & $-1,46$ & $-1,51$ & $-0,93$ & $-0,255$ & $-1,51$ \\
\hline $\mathbf{3}$ & $-1,46$ & $-1,89$ & $-1,14$ & $-0,126$ & $-1,89$ \\
\hline $\mathbf{3 , 5}$ & $-1,18$ & $-2,3$ & $-1,36$ & $-0,255$ & $-2,3$ \\
\hline $\mathbf{4}$ & $-1,17$ & $-2,76$ & $-1,36$ & $-0,389$ & $-2,76$ \\
\hline $\mathbf{4 , 5}$ & $-0,435$ & $-3,27$ & $-1,59$ & $-0,527$ & $-3,27$ \\
\hline $\mathbf{5}$ & $-0,212$ & $-3,85$ & $-1,6$ & $-0,389$ & $-3,85$ \\
\hline
\end{tabular}

Tabela 4 - Medidas de Intensidade Luminosa vs distância para a solução de $\mathrm{KMnO}_{4}$ 


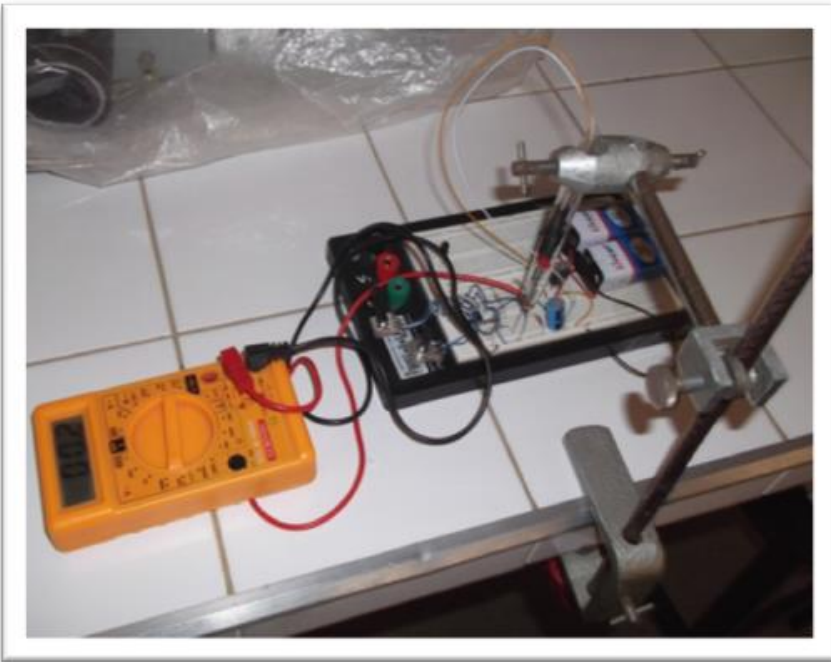

Figura 1- Fotômetro didático desenvolvido no laboratório da FAFISM.

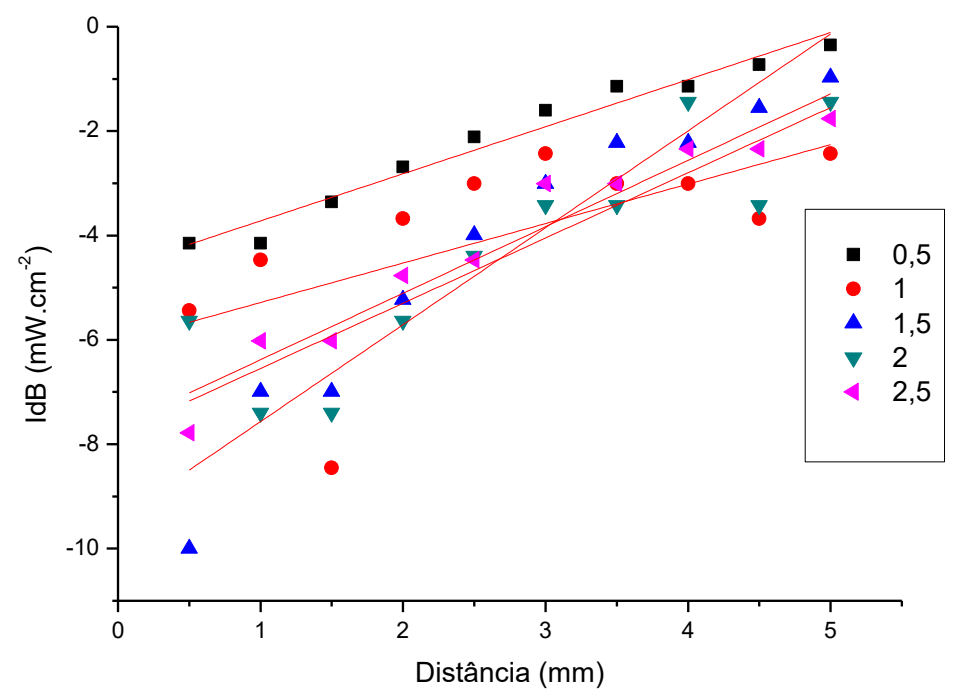

Figura 2 - Intensidade Luminosa $v s$ distância para diferentes concentrações de $\mathrm{CuSO}_{4}$ 


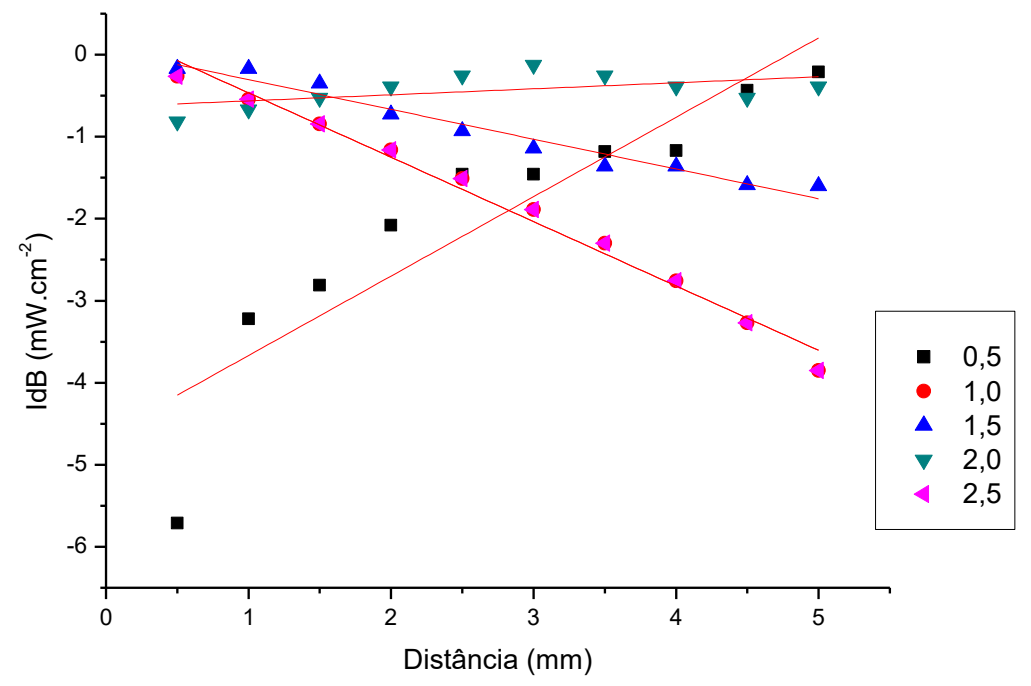

Figura 3 - Intensidade Luminosa vs distância para diferentes concentrações $\mathrm{KMnO}_{4}$

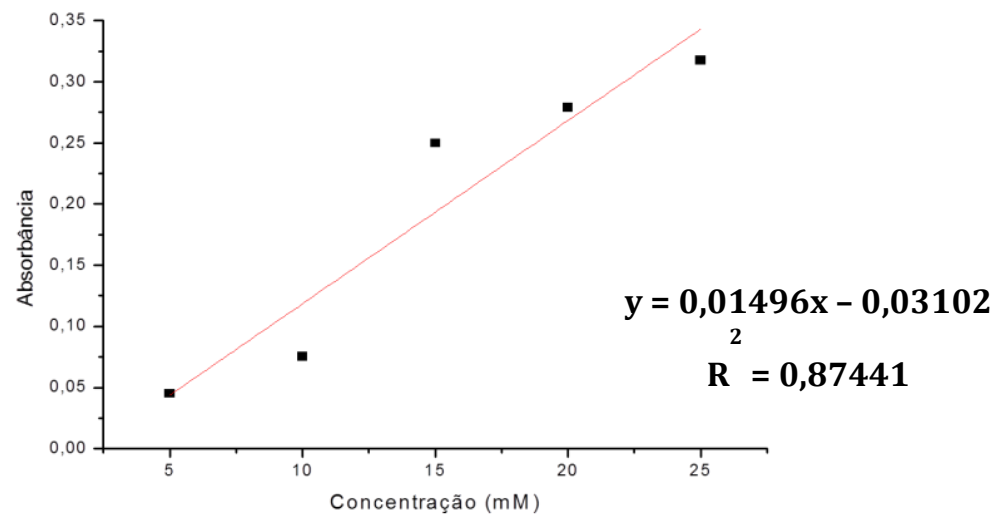

Figura 4- Dados Experimentais para a espectroscopia do $\mathrm{CuSO}_{4}$ para diferentes concentrações molares utilizando o LED emissor verde 


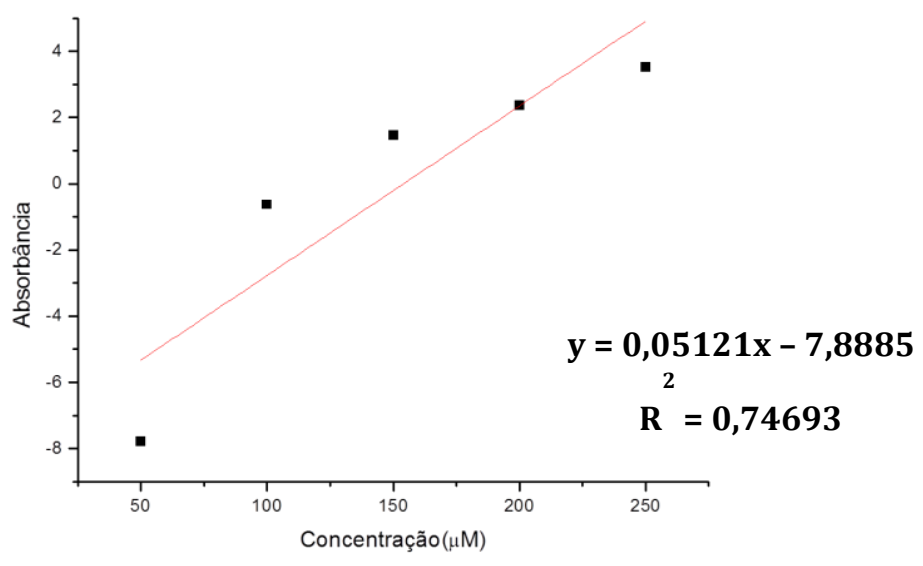

Figura 5- Dados Experimentais para a espectroscopia do $\mathrm{KMnO}_{4}$ para diferentes concentrações molares utilizando o LED emissor vermelho. 